unvereinbar. Stucki ${ }^{56}$ hat für den deutschsprachigen Raum überzeugend gezeigt, dass Tiere aus rechtstheoretischer Sicht ohne weiteres Rechtssubjekte und Träger von Grundrechten sein können. Unrealistisch? Indiskutabel? Nein, denn unsere Gesellschaft hat sich - aus gutem Grund - historisch gesehen stets als wandlungsfähig erwiesen und zwar gerade auch im Zusammenhang mit der Etablierung neuer Rechtspositionen von Schwächeren und Diskriminierten. Fraglos ist die Hürde für eine allgemeine Akzeptanz einer grundsätzlichen Änderung unseres Umgangs mit Tieren besonders hoch. Es geht um nichts Geringeres als die Überwindung der Speziesgrenze. Mit der Anerkennung eines Rechts auf Leben sowie körperliche und geistige Unversehrtheit, eines Recht aufBewegungsfreiheit, eines Verbots der Folter und der grausamen Behandlung und der Abschaffung des Eigentumsstatus für
Tiere wäre zweifellos ein Paradigmenwechsel im Umgang unserer Gesellschaft mit Tieren verbunden. Auf juristischer Ebene ist die Tierschutzverbandsklage derzeit wohl das aussichtsreichste Instrument, um in unserer Gesellschaft Schritt für Schritt einen Bewusstseinswandel hinsichtlich des Umgangs mit Tieren in Gang zu setzen. ${ }^{57}$ Es bleibt zu hoffen, dass die nach dem BlnTSVK anzuerkennenden Tierschutzund Tierrechtsorganisationen das Tierschutzverbandsklagerecht in diesem Sinne erfolgreich nutzen können.

56) Stucki, Grundrechte für Tiere, 2016, S. 293; im Ergebnis ebenso Raspé, Die tierliche Person, 2013, S. 282f; Kloepfer, NuR 2016, 729, 733

57) Hager, NuR 2016, 831, 835.

\title{
Zu Inhalt und Konzeption eines revidierten Landwirtschaftsgesetzes
}

\author{
Zugleich Replik auf die Erwiderung von Czybulka/Fischer-Hüftle/Hampicke/Köck/Martinez, \\ NuR 2021, 227 und 297 \\ Christian Busse
}

( ) Der/die Autor(en) 2021. Dieser Artikel ist eine Open-Access-Publikation.

Der Verfasser hat in der vorliegenden Zeitschrift die „Leipziger Erklärung" des Deutschen Naturschutzrechtstages vom 25. 4. 2018 einer kritischen Analyse unterzogen. Darauf erwiderten Czybulkal Fischer-Hüftle/Hampicke/Köck/Martinez ebenfalls in dieser Zeitschrift. Die Erwiderung nimmt der Verfasser zum Anlass, zum einen seine kritische Analyse zu überprüfen und zum anderen auf einige mit der Debatte verbundene rechtssystematische Fragen einzugehen. Die Debatte fügt sich dabei in die aktuelle Diskussion über die zukünftige Ausgestaltung des Agrarumwelt- und Agrarförderrechts ein.

\section{Drei voneinander zu trennende Fragen}

Die „Leipziger Erklärung“ hat das gegenwärtige Landwirtschaftsgesetz des Bundes (BLwG) als „Anachronismus“ und ,,in seiner ursprünglichen Ausrichtung als ,Einkommensversprechen' völlig überflüssig" bezeichnet. An dessen Stelle müsse ein ,,,echtes' Landwirtschaftsgesetz“ treten, „das Definitionen, Ziele und die Rahmenbedingungen für die zukünftige Landwirtschaftspolitik in Deutschland festlegt". Damit sind gleich drei Fragestellungen angesprochen, die sich inhaltlich und systematisch voneinander trennen lassen. Erstens ist das jetzige BLwG dahinhingehend zu betrachten, ob es Regelungen beinhaltet, deren Beibehaltung sinnvoll erscheint. Dies setzt die Erfassung des aktuellen Inhalts voraus (II.). $\mathrm{Zu}$ prüfen ist zweitens, ob der für das BLwG vorgeschlagene neue Inhalt zweckmäßig und verfassungsrechtlich

\section{Regierungsdirektor Dr. jur. Christian Busse,}

Bundesministerium für Ernährung und Landwirtschaft;

Lehrbeauftragter an den Rechts- und Staatswissenschaftlichen

Fakultät der Universität Bonn;

Dozent an der Hagen Law School und

an der Bundesakademie für öffentliche Verwaltung, Brühl;

Vorsitzender des Ausschusses für Agrarförder- und

Marktorganisationsrecht der Deutschen Gesellschaft für Agrarrecht; Bonn, Deutschland möglich ist. Hierfür müssen die gewollten Inhalte ermittelt werden (III.). Drittens bleibt zu klären, ob die intendierten neuen Inhalte sinnvoll zu einem BLwG passen (IV.). Nach einer Verfolgung dieser drei Fragestellungen endet der Beitrag mit einer Zusammenfassung und Schlusswürdigung (V.)

\section{Der Inhalt des aktuellen BLwG}

Das BLwG geht gemäß seinem $₫ 1$ Satz 1 Halbsatz 2 und 3 davon aus, dass die Landwirtschaft ,gegenüber anderen Wirtschaftsbereichen“ unter ,naturbedingten und wirtschaftlichen Nachteilen“ leidet und diese Nachteile mit ,den Mitteln der allgemeinen Wirtschafts- und Agrarpolitik" auszugleichen sind. Als ein Mittel wird die Produktivitätssteigerung besonders hervorgehoben. $\$ 5$ Halbsatz 2 BLwG spricht insofern von einer „,nachhaltigen Ertragssteigerung“ im Sinne einer stabilen Erhöhung der Produktivität. Nach $₫ 1$ Satz 1 Halbsatz 1 BLwG besteht das Ziel darin, ,der Landwirtschaft die Teilnahme an der fortschreitenden Entwicklung der deutschen Volkswirtschaft“ zu ermöglichen und der „Bevölkerung die bestmögliche Versorgung mit Ernährungsgütern zu sichern“. Zum Teilhabeaspekt präzisiert \$1 Satz 2 BLwG: „Damit soll gleichzeitig die soziale Lage der in der Landwirtschaft tätigen Menschen an die vergleichbarer Berufsgruppen angeglichen werden." \$4 Satz 2 Halbsatz 2 BLwG führt zu dem für die Landwirtschaft geltenden Vergleichsmaßstab aus, dass ,im Wesentlichen von Betrieben mit durchschnittlichen Produktionsbedingungen auszugehen“ sei, „die bei ordnungsgemäßer Führung die wirtschaftliche Existenz einer bäuerlichen Familie nachhaltig gewährleisten“.

Die Teilhabe der in der Landwirtschaft tätigen Menschen an der allgemeinen Wohlstandsentwicklung ist nach Ansicht des Verfassers weiterhin ein erstrebenswertes Ziel. Auch die

1) Deutscher Naturschutzrechtstag, Leipziger Erklärung v. 25.4.2018, ZUR 2018, 469, 469. 
bestmögliche Versorgung der deutschen Bevölkerung mit Nahrungsmitteln dürfte nach wie vor eine sinnvolle Zielsetzung sein. Nur das Mittel der Produktivitätssteigerung scheint, wenn es als Produktivitätserhöhung der Landwirtschaft insgesamt verstanden wird, nicht mehr angemessen, da erstens ausreichend Nahrungsmittel erzeugt werden und zweitens mit einer Erhöhung der Produktivität tendenziell die Umweltbelastung steigt. Der Verfasser hat daher in seiner kritischen Analyse den Aspekt der Produktivitätssteigerung als überholt eingestuft und zugleich seiner Verwunderung Ausdruck darüber gegeben, dass die „Leipziger Erklärung“ das BLwG insgesamt und damit auch die Zielsetzungen der Teilhabe und der Versorgungssicherheit verwirft. ${ }^{2}$

Wird die Erwiderung ${ }^{3}$ zu diesem Punkt betrachtet, so äuBert sie sich bis auf die Zustimmung, dass das Mittel der Produktivitätserhöhung überholt ist ${ }^{4}$, nicht ausdrücklich dazu. Allerdings verweist sie als ,exemplarisches“ Vorbild für einen neu gestalteten Zielekatalog in einem revidierten BLwG auf die im schweizerischen Landwirtschaftsgesetz von 1998 (SchweizLwG) enthaltenen Zielsetzungen. ${ }^{5}$ Dort finden sich allerdings die beiden Zielsetzungen des BLwG wieder. In Art. 1 Buchstabe a SchweizLwG steht an erster Stelle des - in der Erwiderung wörtlich zitierten ${ }^{6}-$ Zielekataloges die ,,sichere Versorgung der Bevölkerung“. Die Teilhabe an der allgemeinen Wohlstandsentwicklung wird zunächst indirekt von zwei Zielsetzungen verfolgt. So fordert Art. 1 Buchstabe d SchweizLwG die ,dezentrale Besiedlung des Landes" und Art. 2 Satz 2 Buchstabe c SchweizLwG die „sozialverträgliche Entwicklung der Landwirtschaft".

Art. 5 Abs. 1 SchweizLwG geht dann direkt auf den Teilhabeaspekt ein: ,Mit den Maßnahmen dieses Gesetzes wird angestrebt, dass nachhaltig wirtschaftende und ökonomisch leistungsfähige Betriebe im Durchschnitt mehrerer Jahre Einkommen erzielen können, die mit den Einkommen der übrigen erwerbstätigen Bevölkerung in der Region vergleichbar sind.“ Dazu ergänzt Art. 5 Abs. 2 SchweizLwG: „Sinken die Einkommen wesentlich unter das vergleichbare Niveau, so ergreift der Bundesrat befristet Maßnahmen zur Verbesserung der Einkommenssituation." Der Kern der zitierten Bestimmungen, der inhaltlich dem BLwG entspricht, geht bis auf die Fassung des schweizerischen Landwirtschaftsgesetzes von 1951 zurück, was verdeutlicht, dass in der Schweiz bei der Neuformulierung des dortigen Landwirtschaftsgesetzes 1998 die Verfolgung des Teilhabeaspekts nicht als ,, anachronistisch " und damit überflüssig eingestuft wurde. ${ }^{7}$

Des Weiteren führt die Erwiderung den französischen Code rural an. ${ }^{8}$ In dessen aktuellen Fassung finden sich in Art. L1 Abs. 1 Abs. 1 und 3 ebenfalls die beiden Zielsetzungen des BLwG wieder. Insofern scheint die Erwiderung der in der „Leipziger Erklärung“ geäußerten Ansicht, dass BLwG sei insgesamt ,,anachronistisch“, nicht zu folgen, da ansonsten der Verweis auf die Vorbildfunktion der Zielsetzungen insbesondere des SchweizLwG widersprüchlich wäre. Dies wird dadurch erhärtet, dass die Erwiderung im Hinblick auf die Kompetenznorm des Art. 74 Abs. 1 Nr. 17 GG ausdrücklich betont, dass zwar ,die Aufnahme der Ernährungssicherung historisch bedingt war", ihr jedoch vor dem Hintergrund einer, ,zunehmend vulnerablen Welt" nicht ,,jeglicher Anwendungsbereich “ abzusprechen sei. Zugleich meint die Erwiderung allerdings an anderer Stelle, dass die Ziele des BLwG ,zur Bewältigung der aktuellen Probleme nichts" beitragen. ${ }^{9}$

Nur nebenbei sei bemerkt, dass die in der Erwiderung geäußerte Ansicht, es sei von der Kompetenznorm der Ernährungssicherung ,,bislang kein Gebrauch gemacht worden" ${ }^{10}$, nicht zutrifft. Seit 1965 bestehen gesetzliche Regelungen zur Sicherstellung der Ernährung im Rahmen einer Versorgungskrise. Diese Regelungen wurden zuletzt mit dem Ernährungssicherstellungs- und -vorsorgesetz v. 4. 4. 2017 (ESVG) $^{11}$ neugefasst. Nach der zugehörigen Gesetzesbegründung ist das ESVG unter anderem auf Art. 74 Abs. 1 Nr. 17 GG gestützt. ${ }^{12}$ Auf das Ernährungssicherstel- lungsrecht des Bundes hatte der Verfasser bereits in seiner kritischen Analyse hingewiesen. ${ }^{13}$

Wird von den beiden Zielsetzungen zu den im BLwG geregelten Maßnahmen übergegangen, so sieht $₫ 2$ Abs. 1 Halbsatz 1 BLwG eine jährliche statistische Erhebung zur Ertragslage der landwirtschaftlichen Betriebe vor, die mittels einer Stichprobe erfolgt und vom Bundesministerium für Ernährung und Landwirtschaft (BMEL) durchzuführen ist. Hieraus hat die Bundesregierung alle vier Jahre für Bundestag und Bundesrat einen ,Bericht über die Lage der Landwirtschaft" zu erstellen, in dem darzulegen ist, inwieweit ein mit ,den Löhnen vergleichbarer Berufs- und Tarifgruppen entsprechender Lohn für die fremden und familieneigenen Arbeitskräfte“ erzielt wurde, ,ein angemessenes Entgelt für die Tätigkeit des Betriebsleiters" angefallen ist und ,eine angemessene Verzinsung des betriebsnotwendigen Kapitals“ vorlag. Bei der „Anlage, Durchführung und Auswertung der Erhebungen und Unterlagen“" wird nach $\$ 3$ BLwG das BMEL von einem Sachverständigenbeirat beraten. Zusammen mit dem Bericht sind gemäß $\$ 5$ BLwG diejenigen Maßnahmen anzugeben, die zur Erreichung der in $\$ 1$ BLwG genannten Ziele getroffen wurden oder noch getroffen werden sollen. $\$ 6$ BLwG regelt, dass bei finanzträchtigen Maßnahmen die entsprechenden Mittel in den Bundeshaushalt einzustellen sind.

Auch bezüglich der statistischen Erhebung versteht der Verfasser nicht, was daran ,,anachronistisch“" sein soll. Sich ein Bild von der wirtschaftlichen Situation der Landwirtschaft im Vergleich zur gesamtwirtschaftlichen Entwicklung bezüglich der Einkommenslage zu machen, erscheint sinnvoll. In der Veröffentlichung der Daten und einem regelmäßigen Bericht gegenüber den gesetzgebenden Körperschaften vermag der Verfasser ebenfalls keine grundlegenden Probleme zu sehen. Gleiches gilt für die Benennung von Maßnahmen, um ein eventuellen Missverhältnis zwischen der Landwirtschaft und anderen vergleichbaren Berufszweigen auszugleichen. Besteht kein Missverhältnis, bedarf es keiner Maßnahmen. Wird ein Missverhältnis festgestellt, so fordert das BLwG lediglich zu Maßnahmen auf, ohne diese konkret zu benennen. Die Maßnahmen werden nicht auf der Grundlage des BLwG, sondern der einschlägigen Kompetenznormen des Grundgesetzes ergriffen. ${ }^{14}$ Soweit es sich

2) Busse, Ein neues Landwirtschaftsgesetz? - Überlegungen zur „Leipziger Erklärung“ des Deutschen Naturschutzrechtstages vom 25.4.2018, NuR 2019, 807, 809.

3) Die Erwiderung ist in zwei Teilen erschienen: Czpbulka/FischerHüftle/Hampicke/Köck/Martinez, Ein Landwirtschaftsgesetz für Deutschland im Zeichen von Umweltschutz und Biodiversität Notwendigkeit, Funktion und Leitbild, NuR 2021, 227, und dies. Ein Landwirtschaftsgesetz für Deutschland im Zeichen von Umweltschutz und Biodiversität - Zentrale Gesetzesinhalte und Finanzierung, NuR 2021, 297.

4) Czybulka/Fischer-Hüftle/Hampicke/Köck/Martinez (Fn. 3), S. 235.

5) Czybulka/Fischer-Hüftle/Hampicke/Köck/Martinez (Fn. 3), S. 233.

6) Czybulka/Fischer-Hüftle/Hampicke/Köck/Martinez (Fn. 3), S. 233.

7) Vgl. näher Norer, Art. 5 Rdnr. 1 ff., in: ders. (Hrsg.), Landwirtschaftsgesetz (LwG), 2019.

8) Czybulka/Fischer-Hüftle/Hampicke/Köck/Martinez (Fn. 3), S. 233.

9) Czybulka/Fischer-Hüftle/Hampicke/Köck/Martinez (Fn. 3), S. $228 \mathrm{f}$

10) Czybulka/Fischer-Hüftle/Hampicke/Köck/Martinez (Fn. 3), S. 234.

11) BGBl. I S. 772.

12) BT-Drs. 18/10943, S. 18.

13) Busse (Fn. 2), S. 811.

14) Im Gesetzgebungsverfahren ist entsprechend darauf geachtet worden, dass sich aus dem BLwG kein ,,Rechtsanspruch einzelner Betriebe auf die Herbeiführung einer Deckung ihres Aufwandes oder ein Automatismus in der Anwendung der Mittel der allgemeinen Wirtschaftspolitik ableiten lässt ...", wie sich aus der Stellungnahme der Bundesregierung v. 11.6.1955 zu dem aus der Mitte des Bundestages eingebrachten Entwurf eines Landwirtschaftsgesetzes ergibt (abgedruckt in: Nonhoff, Das Landwirtschaftsgesetz - Textausgabe mit Erläuterungen und Materialien, 1956, S. 45ff., 45). 
um gesetzliche Maßnahmen handelt, bedürfen sie des Beschlusses durch die gesetzgebenden Körperschaften. Falls sie mit Finanzmitteln verbunden sind, die der Bund aufzubringen hat, muss ihnen der Haushaltsgesetzgeber zustimmen.

Die Erwiderung weist auf die agrarökonomische Diskussion hin, ob die Art und Weise der Vergleichsrechnung sachgerecht ist, und spricht davon, dass die Vergleichsrechnung ,nach einhelligem Urteil der Wissenschaft“ nur eine „sehr eingeschränkte Aussagekraft“ besitze und „vielmehr mit juristischen Verpflichtungen gerechtfertigt" sei. Für dieses „einhellige Urteil“ wird eine einzige, dreißig Jahre alte Literaturstelle angeführt. Offenbar bezweifelt die Erwiderung, dass ein ,genereller Einkommensrückstand“ besteht, ohne sich mit den Ergebnissen des letzten Berichts von 2019 auch nur ansatzweise auseinanderzusetzen. ${ }^{15}$ Für das Wirtschaftsjahr 2017/18 wird dort als „Einkommensabstand" zwischen einem landwirtschaftlichen Haupterwerbsbetrieb und einem in einem Haupterwerbsbetrieb in der gewerblichen Wirtschaft abhängig Beschäftigten eine Minderentlohnung von drei Prozent ausgewiesen. ${ }^{16}$ Was auf den ersten Blick wie eine Einkommensparität aussieht, relativiert sich jedoch schnell durch die weitere Darstellung des Berichts, dass vierzig Prozent des Einkommens landwirtschaftlicher Haupterwerbsbetriebe aus staatlichen Zuwendungen - darunter siebzig Prozent EU-Direktzahlungen - besteht. ${ }^{17}$ Werden diese staatlichen Zuwendungen abgezogen, besteht eine erhebliche Disparität.

Uber Einzelheiten der Vergleichsrechnung lässt sich sicherlich diskutieren. Das Ergebnis einer erheblichen Disparität wird sich jedoch vermutlich nicht wesentlich ändern. Nur mit beträchtlichen staatlichen Zuwendungen komm der Leiter eines landwirtschaftlichen Betriebes im Durchschnitt auf die mittlere Entlohnung eines abhängig Beschäftigten in der gewerblichen Wirtschaft, wobei in Form eines Betriebsleiterzuschlages berücksichtigt ist, dass es sich im Gegensatz zu dem abhängig Beschäftigten um eine Führungsperson handelt. Die Disparität drückt sich unter anderem in einer stetigen Abnahme der landwirtschaftlichen Betriebe aus. Die verbliebenden Betriebe wachsen demgegenüber, wodurch die Bewirtschaftung tendenziell effizienter wird und sich dadurch Kosten verringern. Zugleich wird der Druck erzeugt, intensiver zu wirtschaften, um die Produktivität und damit die Einnahmen zu erhöhen. Durch die intensivere Bewirtschaftung steigt die Belastung der Umwelt, wie auch die Erwiderung annimmt. ${ }^{18}$ Es ist evident, dass eine Verringerung der staatlichen $\mathrm{Zu}-$ schüsse diese Entwicklung forcieren würde.

Die „Leipziger Erklärung“ sieht vor, dass die EU-Direktzahlungen ,in vollem Umfang in ein Honorierungssystem für nachprüfbare ökologische Leistungen der Landwirtschaft umgewandelt werden". Jeder landwirtschaftliche Betrieb könne anschließend selbst entscheiden, ob er an diesem neuen System teilnimmt oder ,,voll auf die ,marktwirtschaftliche Karte“" setzt. ${ }^{19}$ Die hiermit suggerierte Entscheidungsfreiheit besteht jedoch für die meisten landwirtschaftlichen Betriebe nicht. Sie können einen erheblichen Ausfall ihrer Einnahmen nicht kompensieren, da sie in Konkurrenz zu denjenigen Betrieben stehen, die an dem neuen System teilnehmen und darüber Einnahmen generieren. Faktisch wird ein Zwang erzeugt, an dem neuen System teilzuhaben.

Die Erwiderung geht auf die Frage der Wahlfreiheit nicht ein. Wie genau die bisherige Förderung weiterentwickelt werden soll, bleibt zudem im Unklaren. Der Bereich der Förderung, ,für den nach wie vor die EU zuständig ist", wird zwar ausdrücklich aus der Erwiderung ausgeklammert. Wie sich aus der Bezugnahme auf ,nationale Strategien“ ergibt $^{20}$, gehören dazu aber gemäß der Erwiderung nicht EU-Maßnahmen, die die Mitgliedstaaten durch solche Strategien ausgestalten können. Da zugleich von dem Zustandekommen des derzeit verhandelten Konzeptes für die Gemeinsame Agrarpolitik (GAP) ab 2023 mit seiner nationalen Strategieplanung ausgegan- gen wird ${ }^{21}$, stuft die Erwiderung offenbar einen erheblichen Teil der kommenden GAP-Mittel als für das vorgeschlagene Konzept nutzbar ein. Anders als die „Leipziger Erklärung“ schließt sie allerdings die weitere Gewährung von EU-Direktzahlungen nicht explizit aus, was offenbar daran liegt, dass die EU-Direktzahlungen im Rahmen der kommenden nationalen Strategieplanung nicht vollständig aufgehoben werden können. Zugleich wird gefordert, dass „ökologische und betriebliche Grundanforderungen“, deren Einhaltung gegenwärtig im Rahmen der EU-Agrardirektzahlungen mit entlohnt wird, zukünftig - abgesehen von einer Übergangszeit - nicht mehr förderfähig sind. Der Förderung sollen vielmehr nur „Leistungen der Landwirte für Umwelt und Biodiversität", die über die Grundanforderungen hinausgehen, zugänglich sein. ${ }^{22}$

Der Verfasser hat in seiner kritischen Analyse darauf hingewiesen, dass die „Leipziger Erklärung“ die wirtschaftlichen Rahmenbedingungen außen vor lässt. ${ }^{23}$ Nur eine ökonomisch stabile Landwirtschaft kann den gewünschten Richtungswechsel meistern. Führt der Richtungswechsel dazu, dass ein erheblicher Teil der landwirtschaftlichen Betriebe in größeren Betrieben aufgeht, so stellt dies genau das Gegenteil von der in der Erwiderung gewünschten Re-Regionalisierung der Landwirtschaft ${ }^{24}$ dar. Schon jetzt werden das Wachsen von Holdingstrukturen in der Landwirtschaft und die damit einhergehende Entfremdung der Betriebe von der sozialen Verankerung vor Ort beklagt. Wird zugleich die Landwirtschaft de-intensiviert und dieser Vorgang nur teilweise finanziell kompensiert, weil die De-Intensivierung partiell als grundsätzliche Betreiberpflicht eingestuft wird, geraten die Betriebe gleich doppelt unter Kostendruck. Zum einen müssen sie Investitionen für erhöhte Produktionsanforderungen tätigen und zum anderen können sie weniger produzieren und damit erlösen. Der Ausweg wäre ein beträchtlicher Anstieg der Agrarurerzeugnispreise und damit der Lebensmittelpreise, was jedoch allein schon wegen der Konkurrenz auf dem EU-Binnenmarkt und dem Weltmarkt schwierig bis unmöglich ist. Dieser Konkurrenz müsste daher entgegengetreten werden, indem Deutschland vom EU-Binnenmarkt und dem Weltmarkt durch einen neu errichteten Außenschutz abgeschottet wird. ${ }^{25}$ Dies ist allerdings weder unionsrechtlich noch WTO-rechtlich zulässig. Die Konsequenz wäre zudem für Deutschland ein teilweiser Abbruch des Importes und Exportes im Agrarbereich.

15) Czybulka/Fischer-Hüftle/Hampicke/Köck/Martinez (Fn. 3), S. 232 In diesem Zusammenhang wird die kritische Analyse des Verfassers als die eines ,hochrangigen Vertreters auf deutscher Ebene" eingestuft, was sie entsprechend des Hinweises in der Eingangsfußnote, dass es sich um ,Ansichten rein wissenschaftlicher und persönlicher Natur" handelt, nicht ist und nach den entsprechenden Richtlinien der Schriftleitung dieser Zeitschrift, die im Impressum wiedergeben sind, auch nicht sein darf.

16) BMEL, Agrarpolitischer Bericht der Bundesregierung 2019, 2019, S. 84.

17) BMEL (Fn. 16), S. 89

18) Czybulka/Fischer-Hüftle/Hampicke/Köck/Martinez (Fn. 3), S. 227 und 235 .

19) Deutscher Naturschutzrechtstag (Fn. 1), S. 470

20) Czybulka/Fischer-Hüftle/Hampicke/Köck/Martinez (Fn. 3), S. 304.

21) Czybulka/Fischer-Hüftle/Hampicke/Köck/Martinez (Fn. 3), S. 232

22) Czybulka/Fischer-Hüftle/Hampicke/Köck/Martinez (Fn. 3), S. 304.

23) Busse (Fn. 2), S. 809.

24) Czybulka/Fischer-Hüftle/Hampicke/Köck/Martinez (Fn. 3), S. 234

25) Czybulka/Fischer-Hüftle/Hampicke/Köck/Martinez (Fn. 3), S. 235 springen zu diesem Aspekt auf die EU-Ebene, obwohl sich ihr Konzept eines BLwG nur auf Deutschland bezieht und damit rein nationale Standards setzt. So äußern sie beispielsweise in Bezug auf die von ihnen geforderte „Abstockung der Tierhaltung in Deutschland“, dass ,,die Aufgabe insbesondere der EU darin“ bestehe, ,den Agrarbinnenmarkt gegenüber nicht nachhaltig produzierten Lebensmitteln außerhalb der EU wirksam zu schützen“. 
Der Verfasser möchte damit keineswegs als Gegner einer nachhaltig wirtschaftenden Landwirtschaft verstanden werden, sondern lediglich darauf hinweisen, dass bei allen Umstellungskonzepten stets die ökonomischen Rahmenbedingungen mitbedacht werden sollten, um unerwünschte oder sogar konterkarierende Nebeneffekte zu erkennen und zu vermeiden. Die wirtschaftliche Situation der Betriebe ist dabei ein wesentlicher Punkt. Eine der gewerblichen Wirtschaft in Bezug auf die Einkommensmöglichkeiten ebenbürtige Landwirtschaft trägt wesentlich zur Attraktivität bei, den Beruf des Landwirts fortzuführen oder neu zu ergreifen. Um die Herausforderungen einer Systemumstellung zu meistern, bedarf es eines gut ausgebildeten und motivierten Nachwuchses. Wird das BLwG unter diesem Blickwinkel betrachtet, so ist sein Ansatz, die Einkommensverhältnisse in der Landwirtschaft offenzulegen und gegebenenfalls bei einer negativen Bilanz mit staatlichen Maßnahmen gegenzusteuern, nach wie vor aktuell und gerade derzeit besonders wichtig.

Da das BLwG die Art der staatlichen Maßnahmen nicht festlegt, kann es sich auch um die Honorierung von Gemeinwohlleistungen handeln, so dass der Verfasser in dem jetzigen Inhalt des BLwG - abgesehen von dem überholten Mittel der Produktivitätserhöhung im Sinne einer quantitativen Steigerung - keinen grundsätzlichen Widerspruch zu den Zielen der „Leipziger Erklärung“ und der Erwiderung sieht. Im Übrigen gehören zu dieser Thematik auch die derzeitigen Bemühungen, den Anteil der Landwirte an den Margen in der Agrar- und Lebensmittelkette zu erhöhen. Genannt seien insofern unter anderem die Richtlinie (EU) 2019/633 über unlautere Handelspraktiken in den Geschäftsbeziehungen zwischen Unternehmen in der Agrar- und Lebensmittelversorgungskette vom 17. 4. 2019, die derzeit in Deutschland umgesetzt wird, und die neuen Regelungen der EU-Agrarstatistik über die Ermittlung der Margen in der Agrar- und Lebensmittelkette. Mithin kann durch solche staatlichen Mittel ebenfalls eine ökonomische Stärkung der Landwirtschaft, die etwa auch Auswirkungen auf die Konkurrenz zwischen Landwirten und außerlandwirtschaftlichen Erwerbsinteressenten auf dem landwirtschaftlichen Bodenmarkt hat, verfolgt werden. Welche Formulierungen des BLwG im Detail nach über 65 Jahren $\mathrm{zu}$ aktualisieren sind, soll vorliegend nicht weiter erörtert werden, da es lediglich um Grundsätze geht.

\section{Der Inhalt der Vorschläge}

Der Verfasser hat in seiner kritischen Analyse den Versuch der Ermittlung unternommen, welche genauen Inhalte die „Leipziger Erklärung“ für ein revidiertes BLwG vorsieht. Da die „Leipziger Erklärung“" weder eine konzise Auflistung der betroffenen Regelungsbereiche noch den Entwurf einer Gliederung für ein solches Gesetz - sie spricht nur vage von ,Definitionen, Zielen und den Rahmenbedingungen für die künftige Landwirtschaftspolitik in Deutschland" - enthält, vermochte die kritische Analyse nur zu einzelnen angesprochenen Punkten Stellung zu beziehen. Aufgenommen werden sollen nach der „Leipziger Erklärung" offenbar das Pflanzenschutzrecht, das Düngerecht, die landwirtschaftsbezogenen Regelungen aus dem Bundesnaturschutzrecht und dem Bundesbodenschutzrecht, das Grundstückverkehrsrecht sowie das Agrarförderrecht. ${ }^{26}$

Die in diesem Punkt ausführlicher ausfallende Erwiderung gibt nun die Möglichkeit, die Thematik näher zu betrachten. Zentrale Aussage ist dabei, dass das neue BLwG „durchsetzbare ökologisch ausgerichtete Betreiberpflichten/Grundpflichten in den Bereichen Immissionsschutz, Naturschutz, Bodenschutz, Gewässerschutz, Pflanzenschutz, Düngung, Gentechnik/Saatgut, Tierschutz" enthalten soll: „Es regelt diese zum Teil selbst und enthält im Übrigen dynamische Verweisungen auf die bundes- und landesrechtlichen Konkretisierungen der Betreiberpflich- ten in den umweltmedienbezogenen Regelungen“. Konkret wird gefordert, $\ 5$ Abs. 2 BNatSchG, $\$ 38$ a WHG, das Recht der landwirtschaftlichen Nutztiertierhaltung und das Düngerecht in das neue BLwG zu überführen. Ferner liege eine Aufnahme der Bestimmungen über die Anwendung von Pflanzenschutzmitteln auf landwirtschaftlich genutzten Flächen nahe.

Hingegen sollen ,die planerischen und technischen Anforderungen an Tierhaltungsanlagen im Bauplanungsbzw. Immissionsschutzrecht verbleiben“. Weiterhin werden die Regelung einer Pflicht der Landwirtschaft zur "Anpassung an den Klimawandel“ und zur „Fortbildung“" von Landwirten sowie einer „Entwicklungsplanung für die Sicherung und Stärkung der Biodiversität in der Agrarlandschaft" einschließlich der „Sanierungsplanung" angeführt. Angesprochen finden sich zudem besondere Pflichten für ,ausgewiesene Belastungsgebiete und spezifische Entwicklungsgebiete" und eine klarstellende Regelung zur FFHVerträglichkeitsprüfung in Natura-2000-Gebieten. Erwähnt wird ferner eine Erweiterung der Definition der „landwirtschaftlichen Tätigkeit“ um die „Landschaftspflege", wobei diese Definition dann offenbar horizontal im gesamten deutschen Recht zu gelten hat, da sie mit allgemeinen Erwägungen begründet wird. Außerdem soll die „,nationale Agrarförderung in das Landwirtschaftsgesetz" - gemeint sind nicht nur rein nationale Haushaltsmittel, sondern auch die Umsetzung der EU-Agrarförderung - übernommen werden. Das Flurbereinigungsrecht wird hingegen mangels Bundeskompetenz ausgeschlossen.

Die Aussagen zum Grundstückverkehrsrecht sind interpretationsbedürftig. So wird davon gesprochen, dass ,einheitliche Grundentscheidungen" in einem neuen BLwG "sicherlich auf die Landesebene ausstrahlen“ und dadurch „,inkohärente, zum Teil widersprechende Regelungen und Entscheidungen durch die Verwaltungen und die Gerichte" verhindert würden. Als ein Beispiel werden ,,share deals im Grundstückverkehrsrecht" erwähnt. ${ }^{27}$ Wenn der Verfasser dies richtig versteht, rückt damit die Erwiderung von der in der „Leipziger Erklärung“ vorgesehenen Regelung des Grundstückverkehrsrechts in einem revidierten BLwG ab, indem sie sich lediglich eine Ausstrahlungswirkung des revidierten BLwG auf bestehende und zukünftige grundstückverkehrsbezogene Regelungen erhofft. Dies trägt der Anmerkung des Verfassers Rechnung, dass der Bund keine Kompetenz für das landwirtschaftliche Grundstückverkehrsrecht mehr besitzt und folglich keine Aufnahme in ein BLwG erfolgen kann. ${ }^{28}$ Nicht erläutert wird allerdings, wie genau das Leitbild einer ,nachhaltigen, umweltverträglichen und biodiversitätserhaltenden Landwirtschaft" ${ }^{29}$ auf die share-deal-Problematik einwirken soll, die beinhaltet, dass Anteile an einer Gesellschaft, die über landwirtschaftlichen Grund und Boden verfügt, ohne Genehmigung nach dem Grundstückverkehrsgesetz übertragen werden können. Mit einer Regulierung von share deals soll der Steigerung von Kaufpreisen und Pachtentgelten im Agrarbereich entgegengewirkt werden.

Dass der Bund für die übrigen erfassten Bereiche eine prinzipielle Gesetzgebungskompetenz besitzt, dürfte unstreitig sein. Etwas Anderes hat der Verfasser in seiner kritischen Analyse auch nicht vertreten. Fraglich ist allein der Punkt, ob nach Art. 72 Abs. 3 Nr. 2, 4 und 5 GG Regelungen in den Bereichen Naturschutz, Landschaftspflege, Raumordnung und Wasserhaushalt, die in ein revidiertes BLwG aufgenommen würden, unter dem Abweichungsvorbehalt der Bundesländer stehen. Die „Leipziger Erklä-

26) Deutscher Naturschutzrechtstag (Fn. 2), S. 469 f.

27) Czybulka/Fischer-Hüftle/Hampicke/Köck/Martinez (Fn. 3), S. 233 und $300 \mathrm{ff}$.

28) Busse (Fn. 2), S. 812

29) Czybulka/Fischer-Hüftle/Hampicke/Köck/Martinez (Fn. 3), S. 233. 
rung" hat dazu vertreten, dass durch die Einkleidung der betreffenden Fachregelungen als ,obligatorische Betreiberpflichten im Landwirtschaftsgesetz" der Abweichungsvorbehalt nicht gilt. ${ }^{30}$ Worauf abweichungsfeste entsprechende Betreiberpflichten gestützt werden sollen, erläutert die „Leipziger Erklärung“ nicht. Diesbezüglich hat der Verfasser in seiner kritischen Analyse angemerkt, dass dafür Art. 74 Abs. 1 Nr. 17 GG, auf dessen Aspekten „Förderung der landwirtschaftlichen Erzeugung“ und ,Sicherung der Ernährung" das gegenwärtige BLwG beruht, nicht genutzt werden kann. ${ }^{31}$ Eine - in den Worten der ,,Leipziger Erklärung“ - durch das Bundesrecht gezogene „,klare Grenze ..., die für alle Betriebe verbindlich gilt" ${ }^{\text {"32 }}$, ist damit in einer solcher Absolutheit nicht realisierbar.

Die Erwiderung hält dem entgegen, dass Art. 74 Abs. 1 Nr. 17 GG nach der Rechtsprechung des BVerfG, ,auch eingreifende Regelungen“ gestatte. Hierfür wird auf zwei Entscheidungen des BVerfG zum Weinabgabe sowie zum Wasser- und Bodenverbandsrecht verwiesen. ${ }^{33}$ Beide Bereiche stehen jedoch im Kontext zu einer Förderregelung. Mit der Weinabgabe wird die Förderung der deutschen Weinwirtschaft finanziert. Die Gründung von Wasser- und Bodenverbänden dient der Förderung der Landbewirtschaftung. Auch andere eingreifende Regelungen wie etwa die bundesrechtlichen Durchführungsbestimmungen zur - nicht mehr existenten - EU-Milchquotenregelung sind Bestandteile eines Agrarfördersystems. So war die EU-Milchquotenregelung ein Baustein der EU-Milchmarktordnung, die einen stabilen EU-Milchmarkt gewährleisten soll.

Hingegen kann Art. 74 Abs. 1 Nr. 17 GG nach Ansicht des Verfassers nicht dazu genutzt werden, eingreifende Regelungen, die eindeutig einer anderen Bundeskompetenz zugeordnet werden können, zu erlassen. Hier liegt ein Unterschied $\mathrm{zu}$ dem von der Erwiderung angeführten Fall des Düngerechts ${ }^{34}$, da es für das Düngerecht keine spezielle Kompetenz des Bundes gibt. Die Düngung dient der Produktion und damit der Förderung der Landwirtschaft. So heißt es in $\$ 1$ Nr. 1 und 2 DüngeG und damit an erster Stelle: ,Zweck dieses Gesetzes ist es, 1. die Ernährung von Nutzpflanzen sicherzustellen, 2. die Fruchtbarkeit des Bodens ... zu verbessern ..." Die „Betreiberpflichten" sind hingegen nicht vorrangig zur Förderung der Landwirtschaft, sondern zum Schutz von Umwelt-, Klima- und Tierschutz konzipiert. ${ }^{35}$ Was die Einbettung in eine finanzielle Förderregelung betrifft, so unterscheidet die Erwiderung zwischen nicht ausgleichspflichtigen Betreiberpflichten und Betreiberpflichten, für die es eine finanzielle Kompensation geben soll. ${ }^{36}$ Für die erste Kategorie scheidet eine Einordnung als Förderregelung per se aus.

Für die zweite Kategorie ließe sich eine solche Einordnung vertreten, wenn die Pflichten primär mit dem Zweck einer Förderung verbunden wären. Indes sollen sämtliche Betreiberpflichten ordnungsrechtlich flankiert werden und selbständig durchsetzbar sein ${ }^{37}$, so dass sie keinen originären Bestandteil eines Fördersystems bilden. Es handelt sich vielmehr um Vorgaben im jeweiligen Fachrecht, die - wie in der bisherigen Cross-Compliance-Regelung der EU-Agrardirektzahlungen ${ }^{38}$ - im Förderrecht in Bezug genommen werden. Dies wird auch darin deutlich, dass gemäß der Erwiderung bestehende Regelungen des Bundesnaturschutz- und Bundeswasserhaushaltsrechts in das novellierte BLwG überführt werden sollen. Das Etikett eines BLwG und die Ummantelung als „Betreiberpflichten“ für einen landwirtschaftlichen Betrieb führen nicht dazu, anderweitig geregelte Bundeskompetenzen unter Art. 17 Abs. 1 Nr. 17 GG fassen zu können. Die Erwiderung ist im Übrigen in diesem Punkt auch nicht stringent, da sie kurz zuvor ausdrücklich auf die ,Abweichungsrechte der Länder ... (Art. 72 Abs. 3 Nrn. 2 und 5 GG) "hinweist. Offenbar möchte sie, wie auch eine zugehörige Fußnote - „So dürfen die Länder sowohl im Bereich des Naturschutzes als auch im Bereich des Gewässerschutzes vom Bundesrecht abweichende Regelungen treffen.“ - belegt ${ }^{39}$, der „Leipziger Erklärung“ gerade nicht folgen. Dies unterstellt wäre sie in diesem Punkt einer Ansicht mit der kritischen Analyse des Verfassers.

Der Aspekt wirft im Hinblick auf die Gesamtkonzeption der Erwiderung eine weitere bedeutsame Frage auf, die die Erwiderung jedoch nicht thematisiert. Wenn die Bundesländer in gewichtigen Punkten der Betreiberpflichten vom Bundesrecht abweichen und die entsprechende Betreiberpflicht sogar - vorbehaltlich entgegenstehendem EU-Recht - ganz aufheben dürfen, ist es nicht möglich, ein bundeseinheitliches Betreiberpflichtenkonzept zu gewährleisten und ordnungsbehördlich durchzusetzen. Daran schließt sich an, wie mit einer bundesrechtlich geregelten Förderung $\mathrm{zu}$ verfahren ist, die an die Einhaltung einer davon betroffenen Betreiberpflicht geknüpft ist, wenn die Betreiberpflicht durch Landesrecht geändert oder aufgehoben wird.

Nur nebenbei sei angemerkt, dass die Erwiderung der Ansicht des Verfassers, der „Förderrahmen für den Agrarbereich" sei ,gegenwärtig durch die EU vorgegeben“40, entgegenhält, dass dies ,,andere Mitgliedstaaten nicht daran gehindert" habe, ,unter Beachtung der unionalen Vorgaben eigenständige Regelungen zu treffen“"41. Welche „,eigenständigen Regelungen" gemeint sind, bleibt unklar, zumal die Erwiderung kein einziges Beispiel aus einem anderen EU-Mitgliedstaat anführt. Im weiteren Verlauf der Erwiderung wird bis auf die schon genannte kurze Erwähnung des Code rural an keiner Stelle auf das Agrarrecht anderer EU-Mitgliedstaaten Bezug genommen. Das schweizerische Landwirtschaftsgesetz ist gerade keine Regelung eines EU-Mitgliedstaates und daher als Beispiel für ,eigenständige Regelungen“ im Rahmen der EU nicht tauglich.

\section{Zur Konzeption eines novellierten BLwG}

Einen Schwerpunkt in seiner kritischen Analyse hat der Verfasser auf konzeptionelle Fragen gelegt. Die meisten dieser Fragen werden in der Erwiderung nicht näher reflektiert, so dass sich insofern der vom Verfasser angeregte ,dichtere Austausch zwischen Agrar- und Umweltrechtlern“"42 noch nicht eingestellt hat. Zur Klarstellung sei wie-

30) Deutscher Naturschutzrechtstag (Fn. 1), S. 469.

31) Busse (Fn. 2), S. 811.

32) Deutscher Naturschutzrechtstag (Fn. 1), S. 469

33) Czybulka/Fischer-Hüftle/Hampicke/Köck/Martinez (Fn. 3), S. 229.

34) Czybulka/Fischer-Hüftle/Hampicke/Köck/Martinez (Fn. 3), S. 229.

35) Sicherlich dient eine umweltschonende Landwirtschaft auch der Landwirtschaft selbst. Bei Czybulka/Fischer-Hüftle/Hampicke/Köck/ Martinez (Fn. 3), S. 306, heißt es, dass eine „nicht nachhaltige Bodenbewirtschaftung eine ökologische und betriebswirtschaftliche Selbstzerstörung“" sei. Dieses Argument ließe sich auf weitere negative Auswirkungen der Landwirtschaft etwa im Bereich des Klimas erweitern. Mit diesem Argument weite Teile des Agrarumweltrechts ungeachtet spezieller Kompetenztitel unter Art. 74 Abs. $1 \mathrm{Nr} .17 \mathrm{GG}$ zu fassen, erscheint dem Verfasser allerdings zu weitgehend und würde insbesondere dem Kompetenzkatalogcharakter des Grundgesetzes nicht gerecht. Mit einer ähnlichen Argumentation könnte das gesamte Wirtschaftsrecht auf Art. 74 Abs. 1 Nr. 11 GG gestützt werden, obwohl es zahlreiche spezielle Kompetenztitel zum Bereich des Wirtschaftsrechts gibt.

36) Czybulka/Fischer-Hüftle/Hampicke/Köck/Martinez (Fn. 3), S. 299 und 304.

37) Czybulka/Fischer-Hüftle/Hampicke/Köck/Martinez (Fn. 3), S. 301

38) Vgl. dazu Busse/Haarstrich, Agrarförderrecht einschließlich EUPrimärrecht, EU-Kartellrecht und EU-Gerichtsbarkeit, 2. Aufl. 2018, S. $161 \mathrm{ff}$.

39) Czybulka/Fischer-Hüftle/Hampicke/Köck/Martinez (Fn. 3), S. 229 und 300 .

40) Busse (Fn. 2), S. 812

41) Czybulka/Fischer-Hüftle/Hampicke/Köck/Martinez (Fn. 3), S. 228.

42) Busse (Fn. 2), S. 812. 
derholt, dass der Verfasser die Vorstellungen der ,Leipziger Erklärung“ aus rechtlichen und konzeptionellen Gründen für ,wenig geeignet“ eingestuft hat, um damit eine Novelle des BLwG zu unternehmen. ${ }^{43}$ Hingegen hat er nicht vertreten, dass ein „,substanzielles Landwirtschaftsgesetz“ generell abzulehnen ist, wie es ihm die Erwiderung nachsagt. ${ }^{44}$ Auch ist er nicht gegen die Zielsetzung, die - in seinen Worten - „Landwirtschaft an die immer drängender Erfordernisse des Umwelt- und Klimaschutzes besser anzupassen". ${ }^{45}$ Es stellt sich lediglich die Frage, ob die vorgeschlagene Konzeption für ein novelliertes BLwG zur Erreichung dieser Zielsetzung eine geeignete Form darstellt.

Werden die von der „Leipziger Erklärung“ und der Erwiderung als Vorbild angeführten Landwirtschaftsgesetze Frankreichs und der Schweiz betrachtet, so handelt es sich um jeweils umfassende gesetzgeberische Werke, die zahlreiche agrarische Regelungsbereiche in sich vereinen. Das Konzept von zwingenden Betreiberpflichten verwirklicht indes keines der beiden Landwirtschaftsgesetze. Jeden landwirtschaftlichen Betrieb wie eine gewerbliche Anlage, die Anlagenbetreiberpflichten unterliegt, zu behandeln, bricht mit dem traditionellen Verständnis der Landwirtschaft als einem von der gewerblichen Wirtschaft bislang unterschiedenen Wirtschaftszweig. Diesen gewichtigen Punkt, der Auswirkungen auf andere Bereiche - genannt seien das Baurecht und das Steuerrecht - besitzt und das Selbstverständnis der Landwirtschaft insgesamt berührt, hat der Verfasser in seiner kritischen Analyse hervorgehoben. ${ }^{46}$ Die Erwiderung beschäftigt sich mit dem Punkt, der sich aus der von ihr vertretenen Konzeption ergibt, nicht näher, sondern meint lediglich ohne Erläuterung, dass die „Parallele zu den Pflichten der Betreiber bestimmter Anlagen“ nicht bedeute, ,dass der Beruf des Landwirts künftig ein Gewerbe ist".47

Im Kern besteht die Idee darin, die bislang verstreut geregelten Umwelt-, Klima- und Tierschutzverpflichtungen, die mit einer landwirtschaftlichen Tätigkeit verbunden sind, an einer Stelle zusammenzufassen und zugleich in ein Gesamtkonzept einzubinden. Nicht ganz klar wird, ob bei einem Verstoß gegen eine oder mehrere Betreiberpflichten nur die jeweils betroffenen Tätigkeiten oder auch das Betreiben des landwirtschaftlichen Betriebes als solches untersagt werden kann. Eine ,präventive Kontrolle“ stellt sich für die Erwiderung als ,,sachgerecht und verhältnismäBig“ dar, wie es im Abschnitt „Durchsetzung der Grundpflichten“" unter der Überschrift „Eröffnungskontrollen“ heißt. Es müsse dabei „,nicht stets“ ein „Genehmigungsvorbehalt" vorgesehen werden. So seien ,,auch (sanktionsbewehrte) Anzeigepflichten“ denkbar. Zugleich wird eine ordnungsbehördliche „Generalklausel“ als „,notwendiges Gegenstück zu den Betreiberpflichten“" vorgeschlagen, mit deren Hilfe ,,die zuständigen Behörden die Einhaltung der Vorschriften überwachen und nach pflichtgemäßem Ermessen die im Einzelfall erforderlichen Maßnahmen treffen (sollen), um deren Einhaltung sicherzustellen“". ${ }^{48}$ Insgesamt scheint das Konzept noch zwischen der Genehmigung landwirtschaftlicher Tätigkeiten und einer aus dem allgemeinen Gewerberecht bekannten Anzeigepflicht zu schwanken. Um sich hiermit näher auseinandersetzen zu können, bedürfte es zunächst weiterer Konkretisierungen. Sinnvoll wäre es, wenn die Autoren der Erwiderung ihre Vorstellungen in einem Gesetzesentwurf niederlegen und dadurch veranschaulichen würden. Deutlich wird allerdings schon jetzt, dass es sich um mehr als eine bloße kompilatorische Überführung bestehender Einzelverpflichtungen der Landwirte handelt.

Mit den Betreiberpflichten verknüpft werden soll wie schon dargelegt offenbar die gesamte bisherige Förderung im Rahmen der EU-Direktzahlungen und zumindest teilweise der Agrarstrukturpolitik, soweit dies EU-rechtlich zulässig ist. Die bloße Tätigkeit als Landwirt und die dabei erforderliche Einhaltung der Betreiberpflichten werden abgesehen von einem Übergangszeitraum als grundsätzlich nicht förderfähig eingestuft. Die Förderung konzentriert sich mithin auf Umwelt-, Klimaschutz- und Tierschutzmaßnahmen, die über die ohnehin geltenden Betreiberpflichten hinausgehen. Zudem ist ein Ausgleich für „,besondere Belastungen“, die den „Landwirte jenseits der allgemeinen Grundpflichten treffen“, vorgesehen. Daneben werden Maßnahmen als förderwürdig erwähnt, „die der zunehmenden Entfremdung von Landwirtschaft und Gesellschaft entgegenwirken", wozu ein Sammelsurium von „Ausbildung, wissenschaftlicher Forschung, Infrastruktur und Kontaktpflege im regionalen Bereich" angeführt wird. ${ }^{49}$ Auf welche Art und Weise beispielsweise die wissenschaftliche Forschung im Agrarbereich eine Maßnahme gegen eine Entfremdung der Landwirtschaft von der Gesellschaft sein soll, erschließt sich indes nicht ganz. Auch für den Förderbereich gilt, dass es für eine weitergehende Bewertung zunächst einer Darlegung bedürfte, welche Bestandteile der äußerst vielfältigen gegenwärtigen Agrarförderung im Einzelnen überführt oder aufgegeben werden sollen.

Insgesamt scheint der Vorschlag dahin zu gehen, dass ein revidiertes BLwG aus zwei Hauptkapiteln besteht, die die ordnungsrechtlichen Betreiberpflichten und eine darauf abgestellte Förderung beinhalten. Die Betreiberpflichten übernehmen zum Teil schon bestehende Regelungen aus anderen Bundesgesetzen und werden zum Teil neu eingeführt. Den beiden Hauptkapiteln vorgeschaltet ist ein Zielekatalog, aus dem sich nicht nur die beiden Hauptkapitel ableiten, sondern der auf das gesamte deutsche Agrarrecht ausstrahlen soll. Die Ziele bilden zugleich , als einheitliche Grundentscheidungen“ ein „Leitbild“. Alle bestehenden agrarrechtlichen Regelungen in Bund und Ländern sollen im Lichte dieser Ziele ausgelegt werden und neu geschaffene Regelungen diesen Zielen folgen: „Der Gesetzgeber muss sich bei der normativen Konkretisierung am Leitbild orientieren, um eine kohärente Regelung zu erzielen. Das Leitbild strahlt jenseits des Umweltrechts auf andere Bereiche aus, die von zentraler Bedeutung für die Landwirtschaft sind: zum einen auf das Agrarzivilrecht wie die Höfeordnung und das Grundstückverkehrsgesetz, zum anderen auf die Rahmenbedingungen für finanzwirksame Leistungen an die Landwirtschaft." ${ }^{\circ 0}$ Bei normenhierarchisch gleichrangigen Bundesgesetzen kann allerdings ein solches Ergebnis nicht über eine rechtliche Verbindlichkeit des Leitbildes für das gesamte Bundesrecht, sondern nur über das Prinzip der Widerspruchsfreiheit der Rechtsordnung erreicht werden. In Bereichen wie dem Grundstückverkehrsrecht, in dem die Bundesländer die ausschließliche Gesetzgebungskompetenz besitzen, sind direkte rechtliche Vorgaben des Bundes nicht möglich.

Der Passus ,,jenseits des Umweltrechts“ weist zugleich auf ein konzeptionelles Problem hin, auf das die Erwiderung nicht eingeht. Bei Betrachtung der rechtlichen Materien, die in das novellierte BLwG einbezogen werden sollen, und des darauf abgestimmten Zielekataloges zeigt sich, dass es sich nicht um ein Landwirtschaftsgesetz im Sinne einer allgemeinen Regelung der Landwirtschaft - sei es als Rahmengesetz oder Vollgesetz - handelt. Es liegt vielmehr ein Agrarumweltgesetz vor, das die bisherigen umweltrelevan-

43) Busse (Fn. 2), S. 812

44) Czybulka/Fischer-Hüftle/Hampicke/Köck/Martinez (Fn. 3), S. 228.

45) Busse (Fn. 2), S. 812; entsprechend ders., Agrarrecht, Rdnr. 23 in: Schulze/Janssen/Kadelbach (Hrsg.), Europarecht - Handbuch für die deutsche Rechtspraxis, 4. Aufl. 2020, S. $1634 \mathrm{ff}$.: „teilweise aktualisierungsbedürftig“.

46) Busse (Fn. 2), S. 812.

47) Czybulka/Fischer-Hüftle/Hampicke/Köck/Martinez (Fn. 3), S. 300 48) Czybulka/Fischer-Hüftle/Hampicke/Köck/Martinez (Fn. 3), S. 301. 49) Czybulka/Fischer-Hüftle/Hampicke/Köck/Martinez (Fn. 3), S. $304 \mathrm{ff}$. 50) Czybulka/Fischer-Hüftle/Hampicke/Köck/Martinez (Fn. 3), S. 233. 
ten agrarrechtlichen Vorschriften unter einem gemeinsamen Dach zusammenführt und einige neue solcher Regelungen hinzufügt, wobei das Umweltrecht in einem weiten Sinne verstanden wird, indem auch das Tierschutzrecht erfasst wird. So spricht die Erwiderung von dem novellierten BLwG als einem „Instrument“, die „Landwirtschaft aktiv durch ein kohärentes Agrarumweltrecht zu steuern". ${ }^{51}$ Mithin ist der Titel „Landwirtschaftsgesetz“, der wie in der Schweiz oder Frankreich eine recht umfassende Regelung landwirtschaftlicher Aspekte impliziert, nicht passend. Das gegenwärtige BLwG entspricht dem zwar auch nicht, ist aber zumindest näher an einer derartigen Vorstellung, da es die Landwirtschaft in toto als Wirtschaftszweig in Abgrenzung zu anderen Wirtschaftszweigen behandelt.

Letztlich wirkt die Konzeption wie ein Herausziehen der landwirtschaftsbezogenen Regelungen aus dem damaligen Entwurf eines Umweltgesetzbuches (UGB). So enthielt das UGB das Wasserhaushaltsrecht, das Naturschutzrecht, das Bodenschutzrecht, das Pflanzenschutzrecht und das Düngerecht. Ein wesentlicher Grund für sein Scheitern war das Projekt einer integrierten Vorhabenplanung, mit der versucht wurde, zahlreiche Aspekte des UGB in ein einheitliches Anlagenrecht einzubinden. Ähnlich ist auch hier der Gedanke, indem die agrarumweltrechtlichen Regelungen in einem gemeinsamen Leitbild und einem daraus abgeleiteten Korsett von Betreiberpflichten münden sollen.

Wenn der Titel „Landwirtschaftsgesetz“ verwendet wird, dann sollte auch die Landwirtschaft umfassend angesprochen werden. Hierzu gehören die wichtigsten Ziele, die mit dem Agrarrecht verfolgt werden. Dazu zählen unter anderem die beiden jetzt schon im BLwG enthaltenen Zielsetzungen. So wollte etwa der Bundesrat 2001/02 bei seinem Antritt für eine Novellierung des BLwG nicht die bisherigen Zielsetzungen aufgeben, sondern um weitere wie den „Verbraucher-, Tier- und Umweltschutz“ ergänzen, wie bereits vom Verfasser in seiner kritischen Analyse dargestellt wurde. ${ }^{52} 2007$ legte eine Arbeitsgruppe der Agrarministerkonferenz den Entwurf eines novellierten BLwG vor, der in seinen $\int \delta 1$ und 2 einen weit gefächerten Zielekatalog unter Einschluss des ,Tier-, Umwelt- und Naturschutzes" sowie des Nachhaltigkeitsaspektes enthielt und in $\$ 3$ den Aspekt der, ,guten fachlichen Praxis in der Landwirtschaft" aufgriff. ${ }^{53}$ Mit diesem Entwurf setzt sich die Erwiderung nicht auseinander. Durch einen solch breiten Zielekatalog würde zugleich das Spannungsfeld zwischen den vielfältigen gesamtgesellschaftlichen Erwartungen an die Landwirtschaft und den ökonomischen Grundlagen der Landwirtschaft deutlich, dass der Gesetzgeber bei seinen Entscheidungen zu berücksichtigen hat. Ein breiter Zielekatalog macht indes nur Sinn, wenn das BLwG materiell nicht lediglich Agrarumweltrecht enthält.

Abgesehen von diesem allgemein-konzeptionellen Problem ist zu fragen, wie sinnvoll es ist, einzelne Bestimmungen - zum Beispiel die in der Erwiderung genannten $\ 5$ Abs. 2 BNatSchG und $\$ 38$ a WHG - aus den bestehenden Umweltgesetzen herauszuschneiden. Denn sie sind aus sich selbst heraus wenig verständlich, da sie den Zusammenhang mit den übrigen Bestimmungen des jeweiligen Gesetzes voraussetzen. ${ }^{54}$ Bei $\$ 5$ Abs. 2 BNatSchG kommt hinzu, dass Abs. 2 auf der allgemeinen landwirtschaftsbezogenen Regelung des Abs. 1 aufbaut. Außerdem würde der Konnex mit den in den Abs. 3 und 4 enthaltenen Bestimmungen zur Forst- und Fischereiwirtschaft zerstört. In ähnlicher Weise käme es bei einer Überführung des Anwendungsrechts zu Pflanzenschutzmitteln in ein BLwG zu einer Unterbrechung des Sachzusammenhangs mit dem sonstigen Pflanzenschutzrecht, auf dem die Anwendungsbestimmungen begrifflich und systematisch aufbauen. Die Konzeption des bisherigen Cross-Compliance, das das Ordnungsrecht im jeweiligen Fachzusammenhang belässt und aus diesem Fachrecht Folgerungen für die Förderung zieht, erscheint dem Verfasser die konzeptionell geeignetere Variante. Das
Fachrecht im Sinne der Erwiderung auszubauen, thematisch in einem Zielekatalog in Bezug zu nehmen und in eine Liste von Betreiberpflichten aufzunehmen, wird dadurch nicht ausgeschlossen.

Aus rechtssystematischer Sicht ist ergänzend anzumerken, dass es das Schicksal des medienbezogenen Agrarumweltrechts ist, $\mathrm{zu}$ verschiedenen Rechtsmaterien zu gehören. Die landwirtschaftliche Dimension des BBodSchG gehört beispielsweise zum Bodenrecht, zum Agrarrecht und zum Umweltrecht. Die entsprechenden Normen könnten daher sowohl in einem Bodenrechtskodex ${ }^{55}$ als gleichfalls in einem Agrarrechtskodex oder einem Umweltrechtskodex ihren Platz finden. Angesichts eine solchen „Kampfes“ um die Verortung der Bestimmungen spricht viel dafür, sie in ihrem bisherigen medienbezogenen Kontext zu belassen, so reizvoll die Kodexidee auch sein mag.

Einem zusätzlichen Problem der von der „Leipziger Erklärung" vorgeschlagenen Konzeption hat die Erwiderung hingegen Rechnung getragen, ohne dazu allerdings Ausführungen zu enthalten. So hatte der Verfasser in seiner kritischen Analyse angemerkt, dass die Konzeption teilweise über die Landwirtschaft hinausgeht und damit den Rahmen eines Landwirtschaftsgesetzes sprengt. Als Beispiele aus der „Leipziger Erklärung“" wurden von ihm die „Schaffung und Wiederherstellung vernetzter Biotopstrukturen“, „großflächige Landschaftspflegemaßnahmen" mit Zielen außerhalb der Landwirtschaft sowie das Europäische Landschaftsübereinkommen genannt. ${ }^{56}$ In der Erwiderung taucht keines dieser drei Beispiele oder ein parallelgehaltene Beispiel mehr auf.

\section{Schlussbemerkung}

Festhalten lässt sich erstens, dass die ökonomische Blindstelle der „Leipziger Erklärung“ auch von der Erwiderung nicht ausgefüllt wird. Welche wirtschaftlichen Konsequenten das verfolgte Konzept hat, bedürfte einer eingehenden Betrachtung. Damit verknüpft ist zweitens, dass der geforderte Wegfall des sich mit der ökonomischen Lage der Landwirtschaft befassenden Inhalts des gegenwärtigen BLwG fragwürdig erscheint. Denn dadurch ginge eine Grundlage für die gebotene ökonomische Betrachtung verloren. Außerdem ist eine solche ökonomische Dimension in den als Vorbildern herangezogenen Landwirtschaftsgesetzen Frankreichs und der Schweiz enthalten.

Drittens bleibt das Konzept der Betreiberpflichten hinsichtlich seiner näheren Ausgestaltung zu vage, um es eingehender würdigen zu können. Die Wortwahl - „Eröffnungskontrolle“, ,Genehmigungsvorbehalt" und Vorsehen einer ordnungsbehördlichen „Generalklausel“ - deutet auf ein wirtschaftsverwaltungsrechtliches System hin, so wie es aus dem anlagenbezogenen besonderen Gewerberecht bekannt ist. Als ein „Vorbild“ für den Bereich der „Pflichten zur Anpassung an den Klimawandel " verweist die Erwiderung ${ }^{57}$ entsprechend auf die in $\$ 3$ Abs. 2 Nr. 2 12. BImSchV geregelte Pflicht zur Erfüllung ,,umgebungsbedingter Ge-

51) Czybulka/Fischer-Hüftle/Hampicke/Köck/Martinez (Fn. 3), S. 300 und 307.

52) Busse (Fn. 2), S. 811

53) BT-Drs. 16/9161 v. 9.5.2008, S. 5.

54) Czybulka/Fischer-Hüftle/Hampicke/Köck/Martinez (Fn. 3), S. 299 gehen auf das Problem nicht ein, sondern führen lediglich im Zusammenhang mit der Frage, ob „Maßnahmen zur Erhaltung und Förderung der Biodiversität" im BNatSchG oder im BLwG geregelt werden sollen, das folgende Argument an: „Landwirte und ihre Fachbehörden nehmen Vorschriften eher ernst, wenn sie aus dem eigenen Fachrecht kommen, was langfristig auch zu einer anderen Denkweise führen kann.“"

55) Vgl. dazu Busse, Soil Steering Law in Germany - A Contribution to the Systematics of Soil Law, IYSLP 2021 (i.E.).

56) Busse (Fn. 2), S. 812.

57) Czybulka/Fischer-Hüftle/Hampicke/Köck/Martinez (Fn. 3), S. 300. 
fahrenquellen“, die einen Bestandteil der ,,allgemeinen Betreiberpflichten" des $\$ 3$ Abs. 112 . BImSchV bildet.

Das vorgeschlagene Konzept ist viertens allein auf das Agrarumweltrecht im weiteren Sinne zugeschnitten. Dies als Basis eines novellierten BLwG zu verwenden, erscheint begrifflich und rechtssystematisch schwierig. Es entspricht auch erneut nicht den beiden angeführten ausländischen Landwirtschaftsgesetzen. Fünftens begegnet das Herausschneiden von agrarumweltrechtlichen Bestimmungen aus bestehenden Umweltgesetzen fachlichen Bedenken. Um dem Landwirt eine Übersicht über die für ihn bestehenden umweltrechtlichen Pflichten in einem revidierten BLwG zu geben, scheint eine entsprechende Liste mit Weiterverweisungen ins Fachrecht geeigneter. Was die Frage der Gesetzgebungskompetenzen des Bundes angeht, so folgt die Erwiderung fünftens zwar der kritischen Analyse des Verfassers in drei Einzelpunkten - Grundstückverkehrsrecht, Abweichungsrecht der Bundesländer und Beschränkung auf landwirtschaftliche Aspekte - und folgt diesbezüglich der „Leipziger Erklärung“ nicht, vertritt jedoch zum einen hinsichtlich der Auslegung des Art. 74 Abs. 1 Nr. 17 GG eine sehr weite Sichtweise, die mit dem Kompetenzgefüge des Grundgesetzes kollidiert. Zum anderen fehlt es bezüglich des Abweichungsrechts der Bundesländer an einer Betrachtung, was dies für das bundeseinheitlich gedachte Konzept der Betreiberpflichten und der an diese Pflichten gekoppelten Förderung mit sich bringt.

Was schließlich achtens den Förderbereich betrifft, so hat der Verfasser bereits in seinen kritischen Analyse auf die kontrovers beurteilte Frage hingewiesen, ob ordnungsrechtliche oder förderrechtliche Maßnahmen das bessere Mittel sind, gewünschte umweltrechtliche Standards zu erreichen, und angemerkt, dass diese Frage der Mittelauswahl nicht mit dem Niveau der verfolgten Standards verwechselt werden sollte. ${ }^{58}$ Die Erwiderung führt dazu lediglich aus, die ,deutsche Landwirtschaft“ sei ,,in den letzten Jahrzehnten ... mit der Aufgabe überfordert" gewesen, eine ,nachhaltige Bodenbewirtschaftung“ $z u$,,definieren und damit auch zu gewährleisten“. 59

Als Konsequenz soll der Landwirtschaft nun - zumindest weitgehend - die Grundstützung entzogen werden, um die dadurch frei werdenden Mittel nach Ablauf einer Übergangszeit der Förderung von Maßnahmen, die prinzipiell über die Betreiberpflichten hinausgehen, zuzuwenden. Bleibt das Mittelvolumen unverändert, würden - wie auch die Erwiderung anmerk $t^{60}$ - beträchtliche Mittel freigesetzt. Dabei ist allerdings zu beachten, dass die Möglichkeiten der landwirtschaftlichen Betriebe, die neue Zahlung für umwelt-, klima- und tierschutzbezogene Gemeinwohlleistungen natur- und betriebsbedingt höchst unterschiedlich sind. Die Zahlungen müssten - wie auch die Erwiderung vertritt $^{61}$ - mit einer Anreizkomponente verbunden werden, da die Leistung lediglich eines Aufwendungsersatzes nicht ausreichten dürfte, um eine großflächige Nutzung der Maßnahmen zu erreichen. Hat jedoch nur ein Teil der Betriebe die Möglichkeit, die neuen Zahlungen in breiterem Umfang in Anspruch zu nehmen, würde es durch die ungleich verteilten „Gewinnmöglichkeiten“ zu erheblichen Wettbewerbsverzerrungen kommen. Solche Wettbewerbsverzerrungen haben sich in den vergangenen zwei Jahrzehnten etwa im Zuckermarkt gezeigt, in dem Deutschland keine gekoppelte Zahlung gewährt, während die anderen großen zuckererzeugenden Mitgliedstaaten eine gekoppelte Zahlung im $\mathrm{Zu}-$ ckerbereich eingeführt haben. ${ }^{62}$ Dies führt zu der anfangs thematisierten ökonomischen Dimension zurück.

Die Erwiderung schließt mit der Bemerkung, dass das BLwG „,nach 66 Jahren in den wohlverdienten Ruhestand“ geschickt und ein neues BLwG dazu genutzt werden sollte, ein ,kohärentes Agrarumweltrecht“ zu schaffen. Dazu sei der ,parlamentarische Gesetzgeber zuletzt auch durch das neue Umsetzungsmodell der GAP verpflichtet, das wohl im Jahre 2023 in Kraft treten wird und von Deutschland eine ein- heitliche, bundesweite Festsetzung der ökologischen Eckpunkte der Landwirtschaft fordert". Es wird daher angeregt, ,die Zeit bis $2023 \mathrm{zu}$ einer parlamentarischen und gesellschaftlichen Debatte über die ordnungsrechtlichen und die finanzwirksamen Rahmenbedingungen einer künftigen biodiversitätserhaltenden und nachhaltigen Landwirtschaft“ $\mathrm{zu}$ nutzen. ${ }^{63}$ Die neuen GAP-Förderregelungen sollen allerdings - wovon auch die Erwiderung ausgeht - ab 1.1.2023 bereits Anwendung finden. Um dies in Deutschland zu erreichen, muss der deutsche nationale GAP-Strategieplan möglichst bis Anfang 2022 bei der Europäischen Kommission eintreffen. Daher sind die erforderlichen bundesgesetzlichen Grundlagen im Frühjahr 2021 in die parlamentarischen Beratungen eingebracht worden. Die Zeit, bis 2023 eine Debatte in dem angeregten Sinne zu führen, existiert mithin nicht.

Dessen ungeachtet sollte die Diskussion über eine kohärente Regelung des Agrarumweltrechts fortgeführt werden. Ein stimmiges Konzept für diesen Regelungsbereich, das auch die ökonomischen und rechtssystematischen Aspekte einschließt, ist erstrebenswert. Die Aufstellung eines agrarpolitischen Leitbildes in Gesetzesform ist eine Herausforderung, wie die Debatten über ein solches Leitbild im Rahmen der bislang gescheiterten Bestrebungen in mehreren Bundesländern, ein neues landwirtschaftliches Grundstückverkehrsrecht unter Einbeziehung unter anderem der sharedeal-Problematik zu schaffen, zeigen. Nicht nur der Umweltseite, sondern auch der Agrarseite ist bewusst, dass eine derartige Diskussion geführt werden muss, um das Verhältnis zwischen Umwelt und Landwirtschaft entsprechend den aktuellen Herausforderungen angemessen auszutarieren.

So hat der Deutsche Bauernverband im April 2021 vorgeschlagen, Art. 20a GG um die Aspekte des staatlichen Schutzes der „Grundlagen der menschlichen Ernährung“ und „,des Klimas“ zu ergänzen. ${ }^{64}$ Damit wird nicht auf der Ebene des BLwG, sondern auf der höherrangigen Ebene des Grundgesetzes angesetzt. Das Für und Wider dieses Vorschlages soll hier nicht diskutiert werden. ${ }^{65}$ Er kann aber als Anlass dienen, über eine Verortung des Spannungsfeldes auf Verfassungsebene nachzudenken. Hierzu sei der die „Landwirtschaft" behandelnde Art. 104 der schweizerischen Bundesverfassung angeführt, auf dem der Zielekatalog des schweizerischen Landwirtschaftsgesetzes maßgeblich fußt. Er spricht in Abs. 1 eingangs von einer zugleich ,nachhaltigen und auf den Markt ausgerichteten Produktion“" und stellt anschließend in Buchstaben a und b die ,sichere Versorgung

58) Busse (Fn. 2), S. 813

59) Czybulka/Fischer-Hüftle/Hampicke/Köck/Martinez (Fn. 3), S. 306.

60) Czybulka/Fischer-Hüftle/Hampicke/Köck/Martinez (Fn. 3), S. 304 und 306 .

61) Czybulka/Fischer-Hüftle/Hampicke/Köck/Martinez (Fn. 3), S. 299 304 und 306

62) Zu der Ansicht von Czybulka/Fischer-Hüftle/Hampicke/Köck/Martinez (Fn. 3), S. 230, es gebe ,,(etwas vereinfacht) ... in Deutschland seit 130 Jahren zum ersten Mal keine produktbezogenen Stützungsprogramme mehr", sei angemerkt, dass derzeit - vgl. näher Busse/Haarstrich (Fn. 38), S. 34 ff. - produktbezogene EUfinanzierte Förderprogramme in den Bereichen Bienen, Hopfen, frisches Obst und Gemüse sowie Wein existieren. Ferner sei das Schulernährungsprogramm der EU erwähnt. Für die neue GAP-Förderperiode ab 2023 sehen $\int \$ 22$ ff. des Entwurfs eines GAP-Direktzahlungen-Gesetzes (abrufbar über die Internetseite des BMEL) auf Wunsch der Bundesländer eine ,gekoppelte Einkommensstützung “ für Mutterziegen, -schafe und -kühe vor. Die Gesetzesbegründung führt für diese Abkehr von dem seit 2005 in Deutschland befolgten Grundsatz, die EU-rechtlichen Optionen für gekoppelte Direktzahlungen nicht zu nutzen, unter anderem den „Umwelt- und Artenschutz“ an.

63) Czybulka/Fischer-Hüftle/Hampicke/Köck/Martinez (Fn. 3), S. 307.

64) Deutscher Bauernverband, DBV-Zukunftskonzept - Eine neue Partnerschaft für Ernährung und Landwirtschaft, April 2021, S. 3.

65) Zustimmend Martinez, Der „Schutz der Grundlagen der menschlichen Ernährung“ als Staatsziel, AUR 2021, 161. 
der Bevölkerung“ neben die „Erhaltung der natürlichen Lebensgrundlagen und die Pflege der Kulturlandschaft". Im Grundgesetz stehen die beiden auch von der Erwiderung hervorgehobenen Aspekte der Ernährungssicherheit und des Schutzes der natürlichen Lebensgrundlagen mit Art. 20a und Art. 74 Abs. 1 Nr. 17 GG weit auseinander und lassen zudem als Staatszielbestimmung einerseits und Kompetenzzuweisungsnorm andererseits anders als in der schweizerischen Bundesverfassung keine direkte Beziehung zueinander erkennen. Die von der Erwiderung hervorgehobene und als regelungsbedürftig angesehene Funktion des Landwirts als Landschaftspfleger ${ }^{66}$ taucht anders als in der schweizerischen Bundesverfassung im Grundgesetz in Verbindung mit der Landwirtschaft überhaupt nicht auf.

Wird zum Schluss noch einmal zum BLwG zurückgekehrt, so sollte ein solcher Gesetzestitel nach Ansicht des Verfassers einem Gesetz vorbehalten bleiben, das die Landwirtschaft nicht nur unter dem Aspekt eines Sachbereichs, sondern breiter angelegt erfasst. Insofern können das SchweizLwG und der Code rural, aber zum Beispiel auch das Bayerische Agrarwirtschaftsgesetz als Anschauungsmaterial dienen. Wie weitgehend in einem solchen Gesetz das bestehende Agrarrecht zusammengefasst sowie integrierter ausgestaltet wird und in welchem Umfang der Bereich des ländlichen Raumes mit zu erfassen ist, wäre näher zu ergründen. Hierfür kann auf den Optionen, die in dem Bericht von 2008 zur Revision des BLwG dargestellt wurden, aufgebaut werden.

Die Überführung von Bestimmungen, die in einem festen systematischen Kontext anderer Gesetzeswerke stehen, erscheint allerdings prinzipiell problematisch, wie vorliegend anhand des Agrarumweltrechts erläutert wurde. In solchen Fällen empfiehlt sich eher die Nutzung einer Verweisungstechnik. Auch die Aufnahme von Gesetzen, die nicht nur auf die Landwirtschaft anwendbar sind, bleibt schwierig. $\mathrm{Zu}$ nennen sind etwa das Pflanzenschutzgesetz und das Düngegesetz, da sie an erster Stelle die Herstellung und Vermarktung der betreffenden Stoffe regeln und insofern zum Rechtsgebiet des Gefahrstoffrechts gehören. Außerdem ist die Regulierung der Anwendung der Stoffe nicht lediglich auf die Landwirtschaft beschränkt, sondern umfasst auch den privaten Gebrauch.

$\mathrm{Ob}$ im Rahmen eines revidierten BLwG allgemeingültige Definitionen der agrarrechtlichen Grundbegriffe - Land- wirtschaft, Landwirt, landwirtschaftlicher Betrieb und landwirtschaftliche Fläche - geschaffen werden sollten, ist eine reizvolle Frage und müsste eingehender in Bezug auf die verschiedenen Anwendungsbereiche der Grundbegriffe geprüft werden. Ähnlich gelagert ist die Frage der Einführung eines Konzeptes von Betreiberpflichten für landwirtschaftliche Betriebe. Hier bedürfte es zunächst der Klärung, ob damit lediglich eine Zusammenfassung bestehender Pflichten oder aber ein neues Ordnungskonzept gewollt ist, das in die Richtung geht, jeden landwirtschaftlichen Betrieb als eine Anlage zu betrachten, die einer Grundgenehmigung und beständigen Überprüfung, ob die Voraussetzungen der Grundgenehmigung noch gegeben sind, bedarf. Eine knifflige Frage ist zudem die Verzahnung mit dem agrarrechtlichen Landesrecht, das unter anderem das mit dem landwirtschaftlichen Anerbenrecht, dem Grundstückverkehrsrecht, dem Flurbereinigungsrecht, dem Siedlungsrecht und dem nach Art. 72 Abs. 3 GG möglichen Abweichungsrecht wichtige Felder des Agrarrechts berührt. Alles in allem bietet sich hinreichender Stoff für eine weitere spannende Diskussion der Thematik.

Open Access. Dieser Artikel wird unter der Creative Commons Namensnennung 4.0 International Lizenz veröffentlicht, welche die Nutzung, Vervielfältigung, Bearbeitung, Verbreitung und Wiedergabe in jeglichem Medium und Format erlaubt, sofern Sie den/die ursprünglichen Autor(en) und die Quelle ordnungsgemäß nennen, einen Link zur Creative Commons Lizenz beifügen und angeben, ob Änderungen vorgenommen wurden.

Die in diesem Artikel enthaltenen Bilder und sonstiges Drittmaterial unterliegen ebenfalls der genannten Creative Commons Lizenz, sofern sich aus der Abbildungslegende nichts anderes ergibt. Sofern das betreffende Material nicht unter der genannten Creative Commons Lizenz steht und die betreffende Handlung nicht nach gesetzlichen Vorschriften erlaubt ist, ist für die oben aufgeführten Weiterverwendungen des Materials die Einwilligung des jeweiligen Rechteinhabers einzuholen

Weitere Details zur Lizenz entnehmen Sie bitte der Lizenzinformation auf http://creativecommons.org/licenses/by/4.0/deed.de.

Open Access funding enabled and organized by Projekt DEAL.

66) Czybulka/Fischer-Hüftle/Hampicke/Köck/Martinez (Fn. 3), S. 303.

\section{Kastenstandhaltung und kein Ende}

\section{Claudia Schürmeier}

(C) Der/die Autor(en) 2021. Dieser Artikel ist eine Open-Access-Publikation.

\begin{abstract}
Seit Jahrzehnten werden Jungsauen und Sauen über Wochen hinweg in Kastenständen zur Zucht von Ferkeln eingepfercht und können sich weder bewegen noch umdrehen. Selbst das Ausstrecken der Beine in Seitenlage wird zum jahrelangen Diskussionsgegenstand, weil aus wirtschaftlichen Gründen um die Breite der Kastenstände und damit um die baulichen Bedingungen in den Ställen gerungen wird. ${ }^{1}$ Die Stimmen in der Gesellschaft, die sich gegen eine solche Behandlung der Schweine, aber auch gegen den in gro-
\end{abstract}

Dr. jur. Claudia Schürmeier, Dipl.-Verw. (FH),

Lehrbeauftragte Fachbereich Verwaltungswissenschaften,

Hochschule Harz,

Halberstadt, Deutschland ßen Teilen nicht artgerechten Umgang mit Tieren in der Landwirtschaft insgesamt wenden, wurden in den vergangenen Jahren immer lauter. Werden politische Entscheidungen zugunsten der Tiere getroffen, sind diese bis zu ihrer Wirksamkeit allerdings oftmals mit langen Übergangsfristen verbunden. Ein Beispiel dafür ist die Beschlussfassung der Siebten Änderungsverordnung zur TierschutzNutztierhaltungsverordnung, die die rechtliche Grundlage für die

1) Schweine sind nicht die einzige Tierart, die zumindest zeitweise in Käfigen gehalten werden. Hier sei beispielhaft nur auf die Käfighaltung von Kaninchen, insbesondere in der konventionellen Kaninchenmast hingewiesen; Hirt/Maisack/Moritz, TierSchG, 3. Aufl. 2016, TierSchNutztV, vor \$31-37, Rdnr. 12 ff. 